exploring design possibilities and technology features will be followed by the formative evaluation of interface design through qualitative and controlled usability studies with target users.

Results In this paper we report on a user centred approach that allows for the successful capture and integration of social science methods to encapsulate user requirements with usability principles in order to develop an effective and user acceptable self-managing STI system.

Discussion and/or Conclusions Working in a cross-discipline collaboration the overall aim is to investigate the impact of user-centred methods in the design of innovative mobile phone and web technology based rapid testing.

\section{P23 'THIS IS A POLICY NOT A PILOT': HOW TO IMPLEMENT ROUTINE OPT-OUT HIV TESTING FOR ACUTE MEDICAL ADMISSIONS IN AN NHS TRUST IN A HIGH PREVALENCE AREA}

doi:10.1136/sextrans-2012-050601c.23

A Barbour, ${ }^{*}$ A Elgalib, S Draper, D R Phillips. Croydon University Hospital, London, UK

Background and Aims Feasibility and acceptability of HIV testing in acute medical settings has been demonstrated in pilot studies. Whereas we report our success of embedding HIV testing in routine clinical care, delivered and sustained by medical staff in an NHS trust.

Methods From July 2011 all patients aged 16-79years, attending the Acute Medical Unit (AMU), have had a standard HIV test unless they decline. Laboratory costs are funded by the local NHS. Literature was produced for staff and patients. Verbal consent and test ordering is carried out by general medical doctors and AMU nurses, all had training by the local HIV team. AMU proformas were updated with sections for HIV test offer and reason for refusal. The HIV team made regular AMU visits to motivate, troubleshoot and feedback progress. From the outset, nurses were more proactive than doctors in applying the policy. Building on their enthusiasm, nurses were empowered so that by October 2011 AMU visits were weaned as nurses took the lead to enforce the policy.

Results 3709 attendees in the first 6 months, median age 57 years, 50\% female, 54.7\% Caucasian, 6\% Black African. 1390 (37.5\%) had samples for HIV testing; their demographic profile is comparable to that for all attendees. HIV test rate went from 33.2\% July-September to $41.3 \%$ October-December $\left(p<0.005 \chi^{2}\right)$. For a random sample $(\mathrm{n}=396)$, HIV test uptake was $84 \%$. Detailed analysis on offer rate and factors associated with uptake will be presented at the conference. Seven new HIV diagnoses were made, all but one had CD4 >200.

Conclusion A routine opt-out HIV testing policy can be delivered by frontline medical staff in an acute setting with no extra resource requirement beyond laboratory costs. Such a policy identifies new HIV patients who would not otherwise have tested and reduces late diagnosis. We believe that policy ownership by nurse champions, with the HIV team as background advisors, is key to achieving and sustaining high test rates.

\section{P24 REGIONAL EXPERIENCE OF INDICATIONS FOR SWITCHING PATIENTS TO RALTEGRAVIR AND MARAVIROC AND SUCCESS AFTER REGIMEN CHANGE}

doi:10.1136/sextrans-2012-050601c.24

E Clarke, * A Bhardwaj, S Sundaram, D Rowen, E Foley, S Samraj. Royal South Hants Hospital, Southampton, UK

Background In small centres there is wide variation in the accessibility of raltegravir (RAL) and maraviroc (MVC) as they are not on the formulary of several trusts. Individual PCT funding is sought on a named patient basis, a time consuming process leading to potential delays in treatment.

Objectives To identify common clinical indications for treatment change to RAL or MVC. To formulate prescribing criteria on the basis of these in order to seek approval from the District Prescribing Committee and streamline treatment provision.

Methods Retrospective case note review of patients started on RAL or MVC attending six HIV clinics within a regional network between 2008 and 2010 and collection of data on reasons for treatment change.

Results 40 patients were reviewed, 37 of whom started RAL and 3 MVC. Multiple factors contributed to therapy change. 22 patients $(55 \%)$ had resistance to other antiretrovirals or treatment failure and $20(50 \%)$ had significant side effects or intolerance to other drug classes. Other factors included cardiovascular risk $(20 \%)$, co-infections (18\%) and co-morbidities (18\%). 14 patients were reviewed for immunological response following treatment. Two were excluded (one unrelated death). Of the remaining 13 patients, $46 \%$ had a suppressed viral load prior to starting RAL, and $92 \%$ were suppressed after 12 months of treatment.

Conclusions This study demonstrates that identifying common indications for switching to RAL and MVC within a regional network supported the process of formulating criteria to prescribe these newer drugs. This unified approach within the region led to improved and timely access to these drugs thereby improving outcomes for patients at small centres. The agreed prescribing criteria were in instances where combination therapy was not possible due to intolerability, side effects, allergies, resistance, drug interactions, co-morbidities, or HIV-2 (for RAL).

\section{P25 EPIDEMIOLOGICAL TREATMENT FOR CHLAMYDIA CO-INFECTION IN MSM WITH A PRESUMPTIVE DIAGNOSIS OF URETHRAL GONORRHOEA IN S. AUSTRALIA}

doi:10.1136/sextrans-2012-050601c.25

C Khaw, ${ }^{*}$ B Li, R Waddell. Royal Adelaide Hospital, Adelaide, Australia

Background Rates of up to $30 \%$ Neisseria gonorrhoeae (NG) and Chlamydia trachomatis (CT) co-infection occur in men. Historically, Men who have Sex with Men (MSM) were considered to have a low incidence of CT. However, increasing prevalence of CT and NG in MSM, especially asymptomatic anorectal infection is a reality. Despite well-established guidelines providing presumptive co-treatment for CT to patients with treatment indications for NG, various centers in Australia differ in their approach to management in MSM. At our Clinic, epidemiological treatment for CT had only been given to heterosexual males with a presumptive diagnosis of urethral gonorrhoea.

Aim This study was to determine if the local prevalence of CT coinfection in MSM justifies epidemiological treatment when a presumptive diagnosis of urethral gonorrhoea is made.

Methods A retrospective review of case notes data, analysed for NG and CT co-infection in male patients was made over a 10-year period from 2001 to 2010. Data analysis was performed using STATA (V.10).

Results The proportion of heterosexual males who were NG smear positive and also found to be CT positive was 33/274 (12\%) CI $8.4 \%$ to $16.5 \%$. The proportion of MSM who were NG smear positive and found to be CT positive as well was 22/207 (13.4\%) CI $9 \%$ to $18.8 \%$.

Conclusion Based on this study, the guidelines at our Clinic were changed. All MSM with a presumptive diagnosis of NG infection are now given epidemiological treatment for CT infection as heterosexual men. 